\section{Brain Metabolic Mapping with MRS: A Potent Noninvasive Tool for Clinical Diagnosis of Brain Disorders}

P.K. Mandal

l:

am excited and honored to introduce this special issue on met-

abolic brain mapping of brain disorders in the American Journal of Neuroradiology. With the global extension of human life expectancy along with various lifestyle and environmental factors, there has been a dramatic increase in the incidence of various central nervous system disorders. These brain disorders with extremely different pathogeneses such as Alzheimer disease (AD), epilepsy, schizophrenia, major depressive disorder, bipolar disorder (BD), and so forth are affecting the quality of life of patients and having an economic impact on their families. The diagnosis is often very difficult and is based on the detection of clinical symptoms, which appear at relatively later stages in the pathogeneses. The efficacy and outcome of treatment regimens are, thus, contingent on diagnostic biomarkers that can facilitate early detection before the onset of clinical symptoms. There has been extensive research on developing surrogate markers for these disorders that can aid in disease detection, prediction of prognosis, treatment evaluation, and development of novel therapeutics.

In recent years, neurochemical and metabolic profiling of the brain through molecular imaging tools like MR spectroscopy and positron-emission tomography have become increasingly important markers of CNS pathologies. PET is an extremely sensitive radionucleotide-based imaging technique that can determine the concentration of specific biomolecules in the picomolar range. With the continuing development of PET methodology and the ever-increasing number of biologically relevant molecular probes, PET has found clinical application in not only disease diagnostics but also drug development as a tool for tracking drug pharmacokinetics. Furthermore, PET imaging has enabled tracking of various crucial neurotransmitter systems.

While PET uses radioligands injected in the bloodstream to image target macromolecules, MR spectroscopy is a noninvasive technique for investigating the biochemical profile of the brain without radioactive tracers. MR spectroscopy has been recently used in a number of clinical studies to supplement conventional MR imaging because it is able to provide the in vivo neurochemical profile of specific brain regions. ${ }^{1} \mathrm{H}$-MR spectroscopy detects several metabolites, including $\mathrm{N}$-acetylaspartate, myo-inositol, choline, lactate, and so forth, in specific brain regions. Similarly, the ${ }^{31} \mathrm{P}-\mathrm{MR}$ spectroscopy technique can be used to obtain wellresolved peaks of inorganic phosphorus, phosphomonoester, phosphodiester, and phosphocreatine and to estimate $\mathrm{pH}$ levels. ${ }^{1}$

- Indicates open access to non-subscribers at www.ajnr.org

http://dx.doi.org/10.3174/ajnr.A4020
Furthermore, development of specialized editing sequences such as MEshcher-GArwood Point REsolved Spectroscopy (MEGA-PRESS) has enabled detection of crucial brain neurochemicals, such as glutathione, that are present at low concentrations. ${ }^{2} \mathrm{MR}$ spectroscopy not only provides in vivo neurochemical characterization of brain pathologies detected by MR imaging, such as tumors, it is also a sensitive indicator of early disease pathology-induced metabolic alterations in brain regions appearing normal on conventional MR imaging. ${ }^{3,4}$ Various animal models and human clinical studies suggest that MR spectroscopy can help in differential diagnoses and can be important in monitoring the effects of therapeutic interventions and evaluating disease outcome. MR spectroscopy has evolved dramatically with the development of novel acquisition and analysis techniques, and it is a time-effective, accurate, and safe method for the estimation of various brain neurochemicals at high spatial resolution. ${ }^{5,6}$ This special issue offers a unique overview of studies that have used in vivo molecular imaging to assess the neurochemical alterations underlying various CNS disorders.

Alzheimer disease is the most prevalent and debilitating neurodegenerative disorder, with current global World Health Organization estimates of approximately 36 million individuals. ${ }^{7}$ Gao and Barker ${ }^{6}$ reviewed the most up-to-date data attesting to the potential of ${ }^{1} \mathrm{H}-\mathrm{MR}$ spectroscopy as a biomarker for mild cognitive impairment $(\mathrm{MCI})$ and $\mathrm{AD}$. The authors reviewed the current methods used to collect MR spectroscopy data and outlined the findings of MR spectroscopy research in AD. They opined an invaluable role for MR spectroscopy as a predictive biomarker for the development of dementia and urged further evaluatory research in this regard. Kantarci ${ }^{8}$ provided a complementary review of the diagnostic value of amyloid-beta $(\mathrm{A} \beta)$ imaging with Pittsburgh compound-B PET in AD. The review highlighted the application of $\mathrm{A} \beta$ imaging, especially in the differential diagnosis of AD.

To assess the clinical applicability of molecular imaging techniques in disease diagnosis, one must compare and validate them with other neuroimaging and behavioral indicators of disease pathology. In an extensive multimodal cross-sectional neuroimaging study on the various structural and metabolic alterations in $\mathrm{AD}$, Carter et $\mathrm{al}^{9}$ examined the extended medial temporal lobe memory network across the spectrum of disease severity from healthy controls to patients with MCI to AD. Their study assessed cognitive function, neurodegeneration with MR imaging morphometry, structural integrity with diffusion tensor imaging, and metabolic alterations with FDG-PET. The authors found significant correlations among fractional anisotropy decrease, gray matter volume, and metabolic alterations, indicating a close association among neurodegeneration, loss of structural integrity, and metabolic impairment, respectively.

Molecular imaging has also proved helpful in tracking brain tumors, due to the unique alterations in their metabolic phenotypes. MR spectroscopy along with MR imaging for tumor classi- 
fication allows detection of these functional metabolic changes, thereby enabling an understanding of the exact nature and stage of brain tumors. Brain tumors have previously been shown to be associated with increased glycine concentration. To assess the potential of glycine as a diagnostic marker for gliomas, Maudsley et $\mathrm{al}^{10}$ quantified glycine in vivo in different subtypes of gliomas by using MR spectroscopy and assessed its relation to other imaging measures, namely diffusion and perfusion MR imaging. They reported escalating glycine concentration only in a subset of gliomas, with significant variability in concentration and distribution. Furthermore, the authors reported that while glycine showed a positive correlation to choline, there was no significant correlation to any diffusion parameters. Dani and Warach ${ }^{11}$ assessed the clinical usefulness of various measures of cerebral metabolism for ischemic strokes. Their review provides comprehensive insight into a multitude of upcoming molecular imaging techniques that hold potential as clinical tools for therapeutic decisions in patients with stroke.

Studies on psychiatric diseases are perhaps the most challenging application of MR spectroscopy because of their heterogeneous nature and uncharacterized pathology. Preliminary molecular imaging studies have shown some early promise in identifying neurochemical abnormalities associated with these disorders. Lee et $\mathrm{al}^{12}$ explored the use of both MR spectroscopy and PET in examining major depressive disorder. Techniques such as ${ }^{1} \mathrm{H}-\mathrm{MR}$ spectroscopy can track amino neurotransmitters such as glutamine and gamma-aminobutyric acid (GABA), which are known to be altered in depression. ${ }^{12}$ Furthermore, ${ }^{19} \mathrm{~F}-\mathrm{MR}$ spectroscopy, which can track fluorinated psychiatric drugs, has good potential for both examining the mechanism of antidepressants and evaluating their treatment efficacy. On the other hand, PET imaging is able to target specific neurotransmitter systems such as the serotoninergic and dopaminergic pathways. The authors concluded that the use of both molecular imaging techniques for exploring the mechanisms underlying depression is timely and worth further research.

Several metabolism alterations have also been detected in psychiatric disorders affecting adolescents, such as schizophrenia, $\mathrm{BD}$, autism spectrum disorder, and attention deficit/hyperactivity disorder (ADHD). MR spectroscopy offers a handle on identifying and tracking these neurochemical alterations in adolescents. $\mathrm{ADHD}$ is the most prevalent neuropsychiatric disorder in children that progresses to adulthood in up to $50 \%$ of the cases. Altabella et $\mathrm{al}^{13}$ reviewed the most recent preclinical and clinical MR spectroscopy studies on ADHD in children and adults. While thus far there have only been a handful of MR spectroscopy studies on ADHD, these have gone a long way in elucidating the different neurochemical alterations underlying ADHD. Particularly, the development of a GABA-editing sequence has allowed investigation into the dysregulation of this important neurotransmitter in ADHD and has presented insight into the underlying neuropathology of the disease. ${ }^{13}$

$\mathrm{BD}$ is another debilitating and often fatal psychiatric disorder that has its onset in adolescence in approximately $65 \%$ of cases. It has been estimated that early-onset BD treatment is often delayed by up to 16 years. As such, research aimed at understanding its underlying pathophysiology and identifying early neuroimaging biomarkers of $\mathrm{BD}$ is critical for improved diagnosis and treatment of pediatric $\mathrm{BD}$. Kondo et al ${ }^{14}$ comprehensively reviewed the MR spectroscopy literature in pediatric $\mathrm{BD}$ and highlighted converging evidence for alterations in the glutamatergic system and mitochondrial dysfunction. The authors concluded that MR spectroscopy can measure glutamate and other neurochemicals relevant to glutamatergic function in vivo and can thus play a pivotal role in future translational studies of pediatric BD diagnosis and treatment.

Given the power of MR spectroscopy as a diagnostic and noninvasive tool in CNS diseases, there have been immense strides in making the technology more clinically viable. The need for determining the neurochemical changes across multiple brain regions in patients with CNS disorders in a clinically viable timeframe has led to developmental of faster 4D echo-planar J-resolved spectroscopic imaging techniques, which combine the advantages of $2 \mathrm{D}$ MR spectroscopic imaging and echo-planar spectroscopic imaging. Sarma et $\mathrm{al}^{15}$ demonstrated $4 \mathrm{D}$ echo-planar J-resolved spectroscopic imaging by using the nonuniform undersamplingbased acquisition and compressed sensing reconstruction and showed it to be a clinically viable imaging technique in patients with obstructive sleep apnea. The study highlights the clinical applicability of their methodology, which could detect additional neurochemical changes in patients with obstructive sleep apnea previously reported by $1 \mathrm{D}$ MR spectroscopy.

Molecular imaging provides an incredible resource for exploring the neuromolecular basis of CNS disorders and the clinical development of both reliable diagnostic biomarkers and targeted therapeutics. However, these molecular imaging tools have not been routinely used for clinical diagnosis, and the existent literature on their use as a diagnostic tool is sparse for most CNS disorders. It has also been difficult to synthesize the available findings into overall molecular models of disease pathology due to the immense methodologic variations among studies, the use of heterogeneous and small sample sizes, and the wide range of brain structures selected for analyses. As such, it is essential to establish standardized protocols for analysis to allow comparison among studies. These imaging modalities are only capable of quantifying a limited number of neurochemicals and can thus only provide microwindows into the molecular pathology of CNS diseases, which often tends to be multifactorial. Nonetheless, extant data imply that these techniques can play an integral role in CNS disorder diagnostics and therapeutics.

Furthermore, the invention of novel acquisition and analysis techniques is continuing to push the scope of molecular imaging as a clinical tool. Development of targeted radioligands for PET imaging is likely to aid in the development of improved therapeutics that result in more personalized and effective treatment regimens. With the advent of high-magnetic-field-strength scanners and advanced spectral-editing techniques, future MR spectroscopy studies are likely to elucidate the role of neurochemical abnormalities in various CNS disorders and aid in refining diagnosis. The aim of this issue is to increase discussion on the potential of molecular imaging as an effective clinical tool for these disorders. We sincerely hope that this collection of articles will inspire further molecular imaging research, particularly toward neurotransmitter and receptor mapping in CNS disorders. 
I am thankful to all the contributors to this special issue. Thanks to Dr Mauricio Castillo, Editor-in-Chief, American Journal of Neuroradiology, for enabling this special issue. I would also like to acknowledge Dr Subrata Sinha, Director, National Brain Research Centre for support. Dr Sumiti Saharan, Research Scientist, is highly appreciated for editorial support.

\section{REFERENCES}

1. Murphy-Boesch J, Stoyanova R, Srinivasan R, et al. Proton-decoupled 31P chemical shift imaging of the human brain in normal volunteers. NMR Biomed 1993;6:173-80

2. Mandal PK, Tripathi M, Sugunan S. Brain oxidative stress: detection and mapping of anti-oxidant marker glutathione in different brain regions of healthy male/female, $\mathrm{MCI}$ and Alzheimer patients using non-invasive magnetic resonance spectroscopy. Biochem Biophys Res Commun 2012;417:43-48

3. Mirowitz SA, Sartor K, Prensky AJ, et al. Neurodegenerative diseases of childhood: MR and CT evaluation. J Comput Assist Tomogr 1991;15:210-22

4. Vion-Dury J, Meyerhoff DJ, Cozzone PJ, et al. What might be the impact on neurology of the analysis of brain metabolism by in vivo magnetic resonance spectroscopy? J Neurol 1994;241:354-71

5. Rossi A, Biancheri R. Magnetic resonance spectroscopy in metabolic disorders. Neuroimaging Clin N Am 2013;23:425-48

6. Gao F, Barker PB. Various MRS application tools for Alzheimer disease and mild cognitive impairment. AJNR Am J Neuroradiol 2014 Apr 17. [Epub ahead of print]
7. Alzheimers Disease International. World Alzheimer report 2009. http://www.alz.co.uk/research/world-report. Accessed June 4, 2014

8. Kantarci K. Molecular imaging of Alzheimer disease pathology. AJNR Am J Neuroradiol 2014 Feb 6. [Epub ahead of print]

9. Carter SF, Embleton KV, Anton-Rodriguez JM, et al. Regional neuronal network failure and cognition in late-onset sporadic Alzheimer disease. AJNR Am J Neuroradiol $2014 \mathrm{Feb} 27$. [Epub ahead of print]

10. Maudsley AA, Gupta RK, Stoyanova R, et al. Mapping of glycine distributions in gliomas. AJNR Am J Neuroradiol 2014 Jan 30. [Epub ahead of print]

11. Dani KA, Warach S. Metabolic imaging of ischemic stroke: the present and future. AJNR Am J Neuroradiol 2014 Apr 10. [Epub ahead of print]

12. Lee TS, Quek SY, Krishnan KR. Molecular imaging for depressive disorders. AJNR Am J Neuroradiol 2014 May 15. [Epub ahead of print]

13. Altabella L, Zoratto F, Adriani W, et al. MR imaging-detectable metabolic alterations in attention deficit/hyperactivity disorder: from preclinical to clinical studies. AJNR Am J Neuroradiol 2014 Jan 30. [Epub ahead of print]

14. Kondo DG, Hellem TL, Shi XF, et al. A review of MR spectroscopy studies of pediatric bipolar disorder. AJNR Am J Neuroradiol 2014 Feb 20. [Epub ahead of print]

15. Sarma MK, Nagarajan R, Macey PM, et al. Accelerated echo-planar J-resolved spectroscopic imaging in the human brain using compressed sensing: a pilot validation in obstructive sleep apnea. AJNR Am J Neuroradiol 2014 Feb 6. [Epub ahead of print] 